\title{
Nanoanalysis in SEM and STEM: EDS Using Silicon Drift Detectors in Comparison to EELS Using Cs-corrected STEM
}

\author{
M. Falke,* R. Terborg,* A. Käppel*, M. Rohde,* M. Gass, ** A. Bleloch**, S. Hermann***, R. \\ Ecke, $* * *$ T. Waechtler***, and S. E. Schulz*** \\ * Bruker-AXS Microanalysis Berlin, Schwarzschildstr. 12, 12489 Berlin, Germany, \\ ** SuperSTEM Laboratory, STFC Daresbury, WA4 4AD, UK \\ *** Chemnitz University of Technology, Center for Microtechnologies, 09126 Chemnitz, Germany
}

Whereas Electron energy loss spectroscopy (EELS) has benefited from careful development effort over the recent decades, energy dispersive X-ray analysis (EDS) is newly on the rise since liquid nitrogen free silicon drift detectors (SDD) became available [1]. After the SEM community accepted the SDDs, now this new technology is being embraced also in TEM. Examples on complementary STEM EELS and SDD-EDS in SEM and TEM will be given.

One example is a feasibility study on reliable and reversible electrical and mechanical flip chip assemblies mediated by coated carbon nanotubes (CNTs) as a connecting material. Bruker SDDs were used for X-ray analysis of the CNTs in SEM and conventional STEM. Additionally, Gatan EELS in a Cs-corrected Nion STEM was carried out. Both, SDD-EDS and EELS revealed the successful $\mathrm{Cu}$ coating of the multiwall carbon nanotubes (MWCNT) utilising atomic layer deposition. The $\mathrm{Cu}$ was found to be partly oxidised. Cs-corrected STEM bright field imaging revealed that carbon sheets wrap around the $\mathrm{Cu}$ and $\mathrm{CuO}$ nanoparticles, incorporating them into the tube walls. Furthermore, SDD-EDS clearly showed the remaining Ni and Co catalyst particles inside the tubes.

The amounts of matter detectable by EELS in a Cs-probe-corrected NION instrument and by EDS in a conventional STEM were evaluated. For EELS the instrument was operated at $80 \mathrm{keV}$, a probe current of about $100 \mathrm{pA}$ in an Angstrom probe and acquisition times of up to 15 min were used. For the EDS probe currents of 200pA in a nm probe, a solid angle for X-ray collection of 0.12 sr and a take off angle of $22.5^{\circ}$ were applied. Because of the less stable conventional instrument, for EDS shorter acquisition times than for EELS were used. Even those short time EDS maps, acquired within one to several minutes only were suitable to investigate the MWCNT outer cover and identify the nm sized Ni and Co catalyst remains within the tubes. In SEM different EDS detector geometries were used to evaluate the detection limit of nm-sized catalyst particles within MWCNTs. Other nmstructures were tested as well.

The study shows, that a combination of SEM- and TEM-spectroscopy techniques can provide reliable data on the $\mathrm{nm}$ and $\mu \mathrm{m}$ scale. A single measurement with $\mathrm{nm}$ and atomic resolution using a high end Cs-corrected instrument with high beam current can serve as a valuable reference for fast successful EDS mapping in conventional S/TEM and SEM on $\mathrm{nm}$ and up to the micrometer scale.

\section{References}

[1] Strüder, et al., Microsc Microanal 4 (1999), 622.

[2] This work was financially supported by the German Federal Ministry of Education and Research (BMBF). the German Research Foundation (DFG) and the UK EPSRC. 
FIG. 1. Left: ordered MWCNT on Si/Tasubstrate in SEM.

Right: the mixed transmission and BSE signal of disordered MWCNTs reveals the where abouts of the catalyst nanoparticles, scale $200 \mathrm{~nm}$.

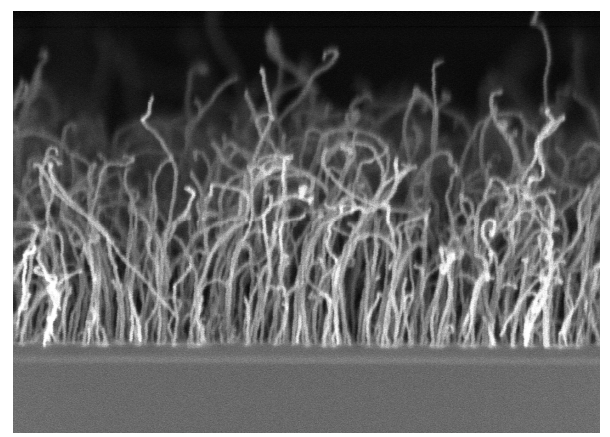

$2 \mu \mathrm{m}$
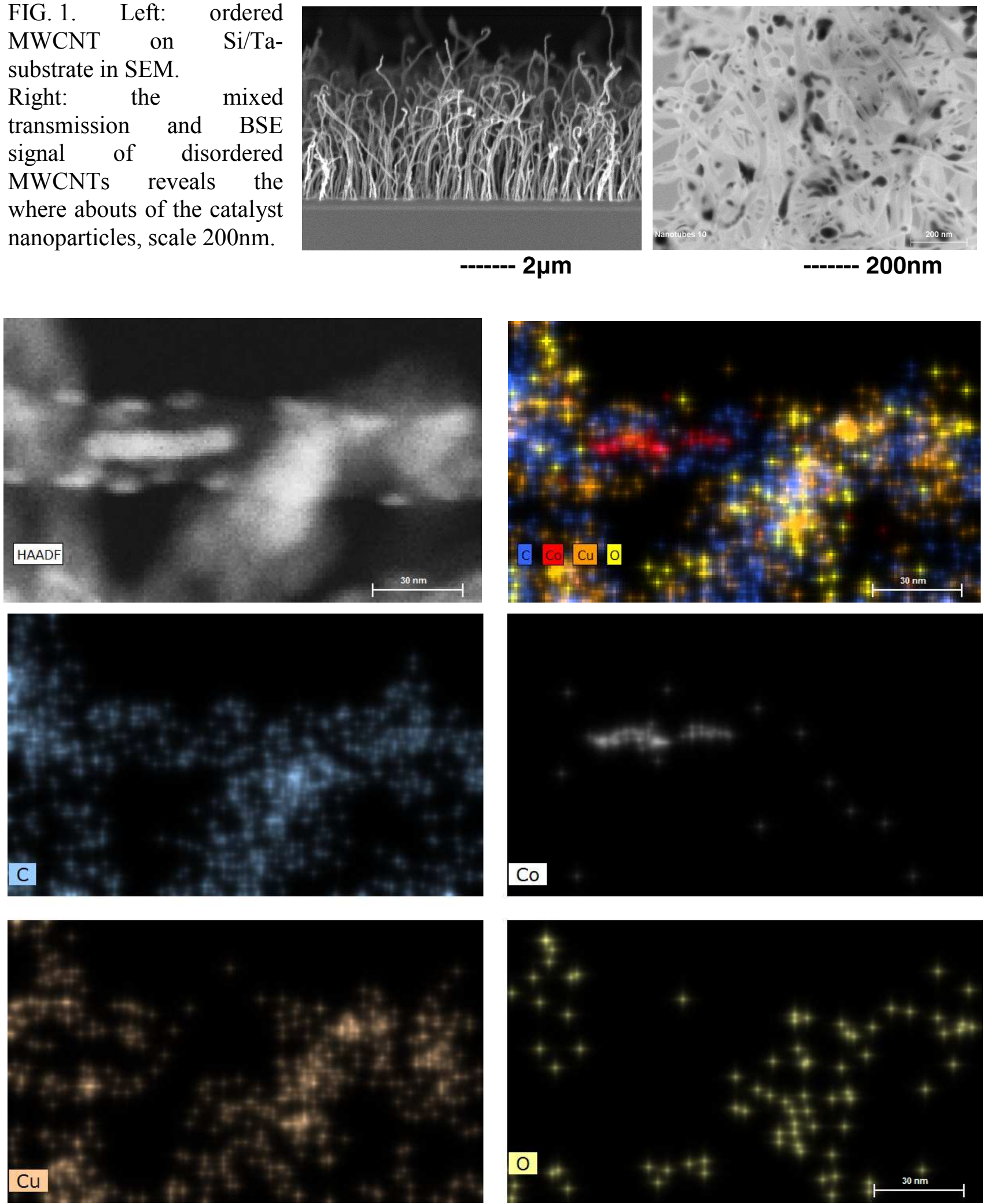

FIG. 2. 1 min element TEM-EDS map of the Cu-coated MWCNTs. A Co-catalyst particle is revealed in the Co map. The successful Cu coating, partly oxidized could be shown as well. 